\title{
SUGERENCIAS PARA EL DESARROLLO DE HABILIDADES EN LA RESOLUCIÓN DE PROBLEMAS
}

\section{Carmen Samper de Caicedo*}

\begin{abstract}
The main purpose of this article is to illustrate some of the phases, of the method proposed by Professor George Pólya, for the resolution of problems, through specific problems. Different ways to approach each phase are shown and problems, directed to students that have not initiated Algebra and that exemplify each aspect, are given.
\end{abstract}

Una de las tareas de los profesores de matemáticas es la de preparar al alumno para resolver problemas. ¿Qué es un problema? Es una situación que representa un reto para la persona que lo enfrenta porque no ve una solución inmediata. Retomando las palabras de George Pólya, problema significa "buscar concretamente una acción para lograr una meta claramente concebida pero no inmediata de alcanzar." La capacidad intelectual del ser humano le permite ser analítico. Esto quiere decir que toda situación que se vive, por más trivial que parezca, es un pequeño problema que se analiza para hallar una solución, tomar una decisión. Estos problemas se resuelven sin pasar por toda la angustia que acompaña la resolución de problemas en la matemática. ¿Por qué la diferencia?

Una situación problemática deja de ser problema si la persona no está interesada en resolverlo. La falta de interés puede estar motivada por alguno de los siguientes aspectos: el tema no es llamativo, no se entiende el problema, no se tienen las herramientas para escoger la estrategia adecuada para resolverlo, no se tienen los conocimientos necesarios para determinar la solución. Nosotros, los profesores de matemáticas, tenemos la oportunidad de retar la curiosidad de nuestros alumnos, proporcionándoles problemas a su nivel, además de las ayudas y métodos para que puedan resolverlos satisfactoriamente.

El Profesor George Pólya, un estudioso de la heurística, el estudio de los métodos y reglas del descubrimiento y la invención, proporciona un método que puede ser usado para la resolución, no sólo de problemas de índole matemático, sino de cualquier tipo de problema. A continuación se resume dicho método.

\section{Método para Resolución de Problemas}

1. Comprensión del problema. Identificar qué es lo que pide el problema y cuál es la información que se necesita para resolverlo.

\footnotetext{
*Profesora Departamento de Matemáticas, Universidad Pedagógica Nacional.
} 
2. Organización de información. Recoger toda la información y organizarla en tablas o gráficas para lograr una mejor comprensión.

3. Plan para resolución. Hacer un dibujo, lista o tabla para buscar patrones, descartar posibilidades o ilustrar la situación. Según el nivel del estudiante, expresar la situación por medio de una ecuación algebraica.

4. Ejecución del plan. Llegar a la solución aplicando la estrategia escogida a la información recocida.

5. Verificación del resultado. Preguntarse si la respuesta tiene sentido, si satisface las condiciones dadas en el problema.

Este método debe comenzar a usarse tempranamente y no cuando el alumno llega al álgebra, ya que los problemas no son solamente de tipo algebraico. Esta preparación debe hacerse en varias etapas. A continuación se darán algunos ejemplos de problemas que pueden ser usados, con alumnos de sexto o séptimo grado de a educación básica, para ir desarrollando la habilidad necesaria en cada uno de los tres primeros pasos del método anterior.

\section{Comprensión del problema}

Se puede comenzar planteando a los alumnos diferentes situaciones problemáticas, para que ellos determinen cuál puede ser el problema, hagan el análisis requerido y propongan caminos para resolverlos. Estas situaciones deben ser de interés general $A$ continuación se enumeran algunas situaciones y varios problemas que pueden surgir de éstas.

1. Situación problemática: El departamento de deportes del colegio necesita comprar todo el equipo para que los alumnos puedan jugar beisbol.

¿Cuál puede ser el problema?

a. En qué consiste el equipo que se desea comprar.

b. De cuánto dinero se dispone.

c. Cómo se puede conseguir más dinero.

2. Situación problemática: Un derrumbe en la tubería que conduce el agua a una ciudad bloqueó el paso de ésta.

¿Cuál puede ser el problema?

a. En que sector de la tubería fue el derrumbe.

b. Qué equipos y cuántos hombres se necesitan para corregir el daño.

c. Cuánto tiempo se demoran en hacer los arreglos.

d. Cómo abastecen a la ciudad de agua durante la emergencia.

e. Cómo pueden los habitantes de la ciudad ayudar mientras dura la emergencia. 


\section{Organización de información}

Es necesario enseñar a los alumnos a interpretar la información que se ha dado, no solamente de forma explícita, sino también en gráficas o tablas, como lo hacen principalmente los medios de comunicación. Ellos deben discernir entre aquellos datos que necesitan para resolver el problema y los que no son pertinentes. Deben determinar qué información hace falta para poder resolver el problema. En este momento, es más importante organizar la información que resolver el problema en sí. En cada uno de los problemas que se presentan a continuación, se da la información de forma diferente.

1. En la siguiente tabla se indica cuál es el uso que se da al agua del Lago de Tota. Escoja la información necesaria de la dada en la tabla, para resolver cada situación mencionada y determine qué otros datos se necesita para resolverla.

\begin{tabular}{|c|c|}
\hline Uso & Litros por segundo \\
\hline Acerías Paz del Río & 200 \\
\hline Poblaciones: Cuítiva & 1.3 \\
Tota & 2.5 \\
Sogamoso & 300 \\
Aquitania & 3.0 \\
\hline Haciendas: A & 120 \\
B & 30 \\
C & 80 \\
D & 70 \\
\hline Regadío de cebolla & 1.000 \\
\hline Evaporación & 1.625 \\
\hline
\end{tabular}

a. ¿Cuántos litros por segundo se sacan del Lago de Tota para uso industrial o agrónomo?

b. ¿Cuántos litros por segundo son para consumo doméstico?

c. ¿En una hora, cuánta agua se evapora?

d. Cada pino consume 9 litros de agua por día. ¿Cuántos litros de agua consume en una hora?

2. Usando el mapa que se muestra a continuación, determine cuál es la información que ayuda a resolver el interrogante y, si hacen falta datos para hallar la solución, diga cuáles son.

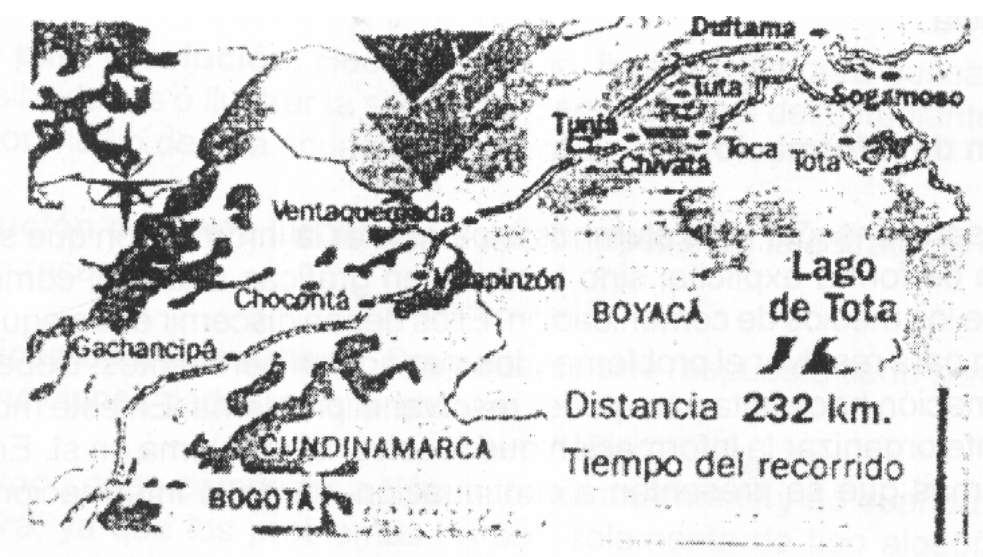

El Tiempo, febrero 15, 1998. 
a. ¿Cuánto tiempo demora un viaje al Lago de Tota desde Santa Fe de Bogotá?

b. ¿Cuántas rutas pueden tomarse para hacer el viaje y. para cada una, por cuáles pueblos se pasa?

3. ¿Cuáles preguntas se pueden contestar con la información dada? Haga una tabla o un dibujo para organizar la información.

Cristina estudia arquitectura y tiene que hacer una maqueta de un edificio. Compró 3 pliegos de cartón paja a $\$ 1400$ el pliego, 2 tubos de pegante a $\$ 2950$ cada uno, in bisturí de $\$ 3240$, 8 varillas de balso, cada una de $90 \mathrm{~cm}$, a $\$ 750$ la varilla, y una lámina de icopor. También compró los siguientes elementos decorativos: 2 docenas de arbolitos, 8 carros, 15 personas y 27 arbustos.

a. ¿Cuánto pagó por el cartón paja y las varillas?

b. Necesita 15 árboles y tres docenas de arbustos. ¿Compró suficiente cantidad de cada uno?

c. ¿Cuánto dinero invirtió para hacer la maqueta?

d. Cristina usó 3/4 de la lámina de icopor. ¿Cuántos centímetros cuadrados de icopor sobraron?

e. Ella empleó 6 1/5 varillas. ¿Cuántos centímetros de balso sobraron?

4. La gráfica muestra el número de metros excavados diariamente por una cuadrilla de obreros de una compañía petrolera. Usando la información dada, responda los interrogantes.

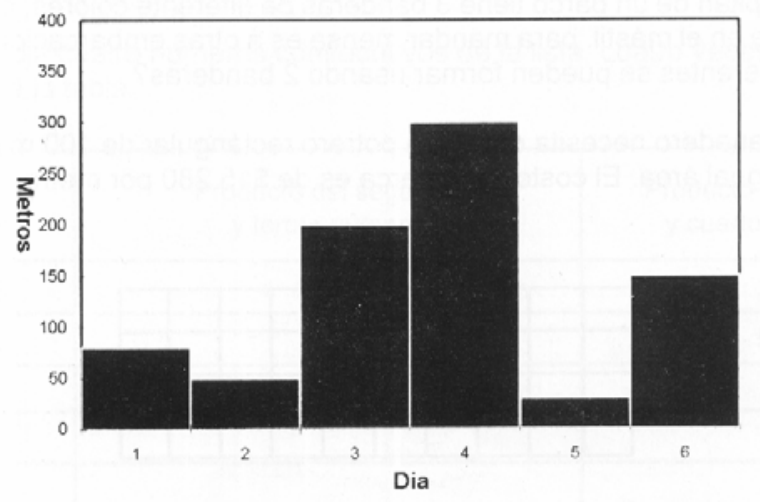

a. Aproximadamente, ¿cuántos metros excavaron en la semana?

b. Cuando el terreno es muy rocoso se dificultan las excavaciones y las brocas se acaban más rápido. ¿Cuál de los siguientes esquemas corresponde al número de brocas usadas?
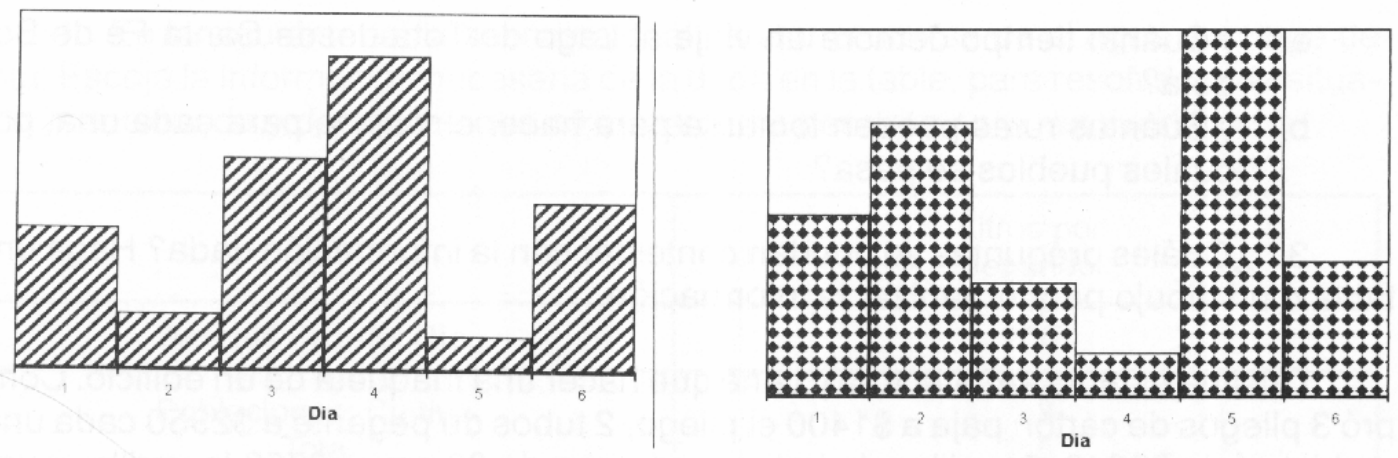


\section{Plan para resolución}

Existen varias estrategias que pueden ser utilizadas en la resolución de problemas. Se pueden hacer listas o tablas, las cuales al analizarlas muestran el patrón que, al generalizarlo, lleva a la solución. Se pueden hacer dibujos y finalmente, se pueden hacer modelos de material manipulable. Todas estas estrategias son pasos previos necesarios para cuando el alumno pueda usar el lenguaje algebraico para escribir ecuaciones o desigualdades que modelen el problema. Por eso es importante que ellos hayan usado estas estrategias anteriormente. A continuación se presentan algunos problemas en los cuales se puede hacer uso de alguna de estas estrategias para resolverlo.

\section{Dibujo}

1. El capitán de un barco tiene 3 banderas de diferenteS colores, que se colocan verticalmente en el mástil, para mandar mensajes a otras embarcaciones. ¿Cuántos mensajes diferentes se pueden formar usando 2 banderas?

2. Un ganadero necesita dividir un potrero rectangular de $100 \mathrm{~m}$ por $40 \mathrm{~m}$ en 4 potreros de igual área. El costo de la cerca es de $\$ 15.280$ por metro.

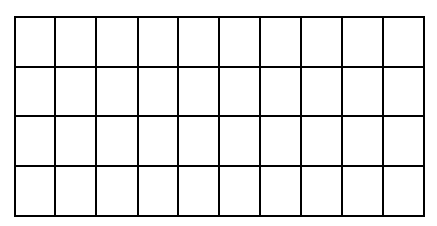

a. Dibuje 5 posibles divisiones del potrero.

b. ¿Para cuál de las posibilidades anteriores es menor el costo de la cerca interna?

c. ¿Cuál es el costo en ese caso?

\section{Lista y tabla}

1. La sucesión de Fibonacci consta de la siguiente lista de números:

$$
1,1,2,3,5,8,13, \ldots
$$

a. ¿Cuál es la regla que se usa para determinar el siguiente número en la lista?

b. Escriba los siguientes tres números de la lista.

c. Escoja tres números consecutivos de la lista, cuatro veces más, y complete la tabla.

\begin{tabular}{|c|c|c|}
\hline Números & Cuadrado del segundo número & Producto del primer y tercer número \\
\hline $1,1,2$ & 1 & 2 \\
\hline $1,2,3$ & 4 & \\
\hline & & \\
\hline & & \\
\hline & & \\
\hline & & \\
\hline
\end{tabular}


Compare los resultados y escriba una conclusión.

d. Escoja cuatro números consecutivos de la lista, cuatro veces más, y complete la tabla.

\begin{tabular}{|c|c|c|}
\hline Números & $\begin{array}{c}\text { Producto del segundo y tercer } \\
\text { número }\end{array}$ & $\begin{array}{c}\text { Cuadrado del primer y cuarto } \\
\text { número }\end{array}$ \\
\hline $1,1,2,3$ & 2 & 3 \\
\hline $1,2,3,5$ & 6 & 5 \\
\hline & & \\
\hline & & \\
\hline & & \\
\hline & & \\
\hline
\end{tabular}

Compare resultados y escriba una conclusión.

2. Comenzando a las 8:00 a.m., un reloj se atrasa 5 minutos cada hora. ¿Cuándo volverá a mostrar la hora correcta?

3. Mauricio salió de Bogotá hacia Ibagué al medio día, manejando su carro a una velocidad promedio de $45 \mathrm{~km} / \mathrm{h}$. A la 1:00 p.m., Rodolfo salió de Bogotá, en la misma dirección, pero él maneja a $60 \mathrm{~km} / \mathrm{h}$.

a) Complete y amplíe la tabla para averiguar a qué hora alcanza Rodolfo a Mauricio.

\begin{tabular}{|c|c|c|c|c|c|c|}
\hline & hora & $\begin{array}{l}\text { Kilometraje } \\
\text { recorrido }\end{array}$ & hora & $\begin{array}{l}\text { Kilometraje } \\
\text { recorrido }\end{array}$ & hora & $\begin{array}{l}\text { Kilometraje } \\
\text { recorrido }\end{array}$ \\
\hline Mauricio & $1: 00$ & 45 & $2: 00$ & & & \\
\hline Rodolfo & $1: 00$ & 0 & $2: 00$ & 60 & & \\
\hline
\end{tabular}

b) Si la distancia entre Bogotá e lbagué es de 204 kilómetros, ¿cuántos kilómetros hacen falta para llegar a lbagué después de encontrarse?

4. La tabla muestra los precios que cobran en el correo, según el peso, para enviar cartas o paquetes dentro del territorio colombiano.

\begin{tabular}{|c|c|}
\hline peso $(\mathrm{g})$ & costo \\
\hline $0-25$ & 500 \\
\hline $26-50$ & 1000 \\
\hline $51-100$ & 1500 \\
\hline $101-250$ & 2000 \\
\hline $251-500$ & 2500 \\
\hline $501-1000$ & 3000 \\
\hline $1001-2000$ & 3700 \\
\hline kilo adicional & 700 \\
\hline
\end{tabular}

Una señora envió 10 cartas y pagó $\$ 10$ 000.00. Dé, por lo menos, 4 combinaciones posibles del número de cartas de cada franja de peso que pudo haber enviado 
5. Complete la tabla para hallar la solución al problema.

La suma de los dígitos de un número de dos dígitos es nueve. El valor del número es nueve veces el dígito de unidades. ¿Cuál es el número?

\section{Material manipulable}

1. Carlota tiene un negocio de pastelería. En una ocasión, le encargaron unas barras de torta de medidas $33 \mathrm{~cm} \times 10 \mathrm{~cm}$. Ella dispone de latas rectangulares de medidas $37 \mathrm{~cm}$ x $87 \mathrm{~cm}$ y de $47 \mathrm{~cm} \times 77 \mathrm{~cm}$. ¿Cuál ata debería usar para que resulten la mayor cantidad de barras y haya el menor desperdicio?

a. Recorte un rectángulo del tamaño de cada lata.

b. Recorte 10 rectángulos de $33 \mathrm{~cm} \times 10 \mathrm{~cm}$. estos representarán las barras.

o. Coloque las barras sobre las latas, primero de tal forma que todas queden en la misma dirección y luego combinando de cualquier forma. En una hoja de papel, ilustre cada patrón que se invente y anote la cantidad que se desperdicia.

d. Responda la pregunta.

2. Invente algún tipo de material que le ayude a resolver el siguiente problema.

Martha tenía unas cajitas selladas, con dos chicles cada una, que compartió con sus amigos. También tenía unas cajas abiertas. A Catalina le da la mitad de las cajas y un chicle de una de las cajas abiertas. A Jairo le da la mitad de las cajas que le quedan y un chicle de las cajas abiertas. A Susana le da la mitad de las cajas que quedan y un chicle. Ella se queda con tres cajas. ¿Cuántas cajas de chicles selladas tenía Martha originalmente y cuántas abiertas?

Dada la edad de los alumnos hacia quienes va dirigida esta propuesta, la estrategia de traducir el problema a una ecuación no es viable. Algunos de los problemas pueden ser resueltos de esa forma, más fácilmente. Sin embargo, el uso de estas estrategias puede mostrarle al alumno cuál es la ecuación necesaria cuando ya tenga las herramientas algebraicas necesarias.

Algunos problemas tienen varias respuestas. Se espera que esto capte el interés de los alumnos porque deben suscitar discusiones. Se construyeron varios de los problemas alrededor de datos que tienen que ver con regiones de nuestro país. El propósito es doble: despertar el interés de los alumnos y aumentar sus conocimientos del país. Para el profesor, son ejemplos de problemas que ellos pueden inventar.

Desarrollar las habilidades anteriores, con problemas no rutinarios, puede hacer el proceso más agradable y significativo. La persona que ha resuelto algún problema matemático siente el triunfo de ser un inventor, un descubridor, y desarrolla el gusto por la matemática.

\section{Bibliografía}

POLYA, George. (1957): How to Salve It. Garden City, NY: Doubleday \& Company, Inc. 